\title{
On the interplay between fast reaction and slow diffusion in the concrete carbonation process: a matched-asymptotics approach
}

\author{
Adrian Muntean
}

Received: 4 July 2007 / Accepted: 13 May 2008 / Published online: 4 June 2008

(C) The Author(s) 2008. This article is published with open access at Springerlink.com

\begin{abstract}
A matched-asymptotics approach is proposed to show the occurrence of two distinct characteristic length scales in the carbonation process. The separation of these scales arises due to the strong competition between reaction and diffusion effects. We show that for sufficiently large times $\tau$ the width of the carbonated region is proportional to $\sqrt{\tau}$, while the width of the reaction front is proportional to $\tau^{\frac{p-1}{2(p+1)}}$ for carbonation-reaction rates with a power law structure like $k\left[\mathrm{CO}_{2}\right]^{p}\left[\mathrm{Ca}(\mathrm{OH})_{2}\right]^{q}$, where $k>0$ and $p, q>1$ and identify the proportionality coefficient asymptotically. We emphasize the occurrence of a water barrier in the reaction zone which may hinder the penetration of $\mathrm{CO}_{2}$ by locally filling with water air parts of the pores. This non-linear effect may be one of the causes why a purely linear extrapolation of accelerated carbonation test results to natural carbonation settings is (even theoretically) not reasonable. Finally, we compare our asymptotic penetration law against measured penetration depths from Bune (Zum Karbonatisierungsbedingten Verlust der Dauerhaftigkeit von Außenbauteilen aus Stahlbeton, 1994). The novelty consists in the fact that the factor multiplying $\sqrt{\tau}$ is
\end{abstract}

A. Muntean $(\varangle)$

Centre for Analysis, Scientific computing and Applications (CASA), Department of Mathematics and Computer

Science, Technical University of Eindhoven, PO Box 513, 5600 MB Eindhoven, The Netherlands

e-mail: a.muntean@tue.nl now identified asymptotically by solving a non-linear system of ordinary differential equations, and hence, fitting arguments are not necessary to estimate its size. We offer an alternative to the (asymptotic) $\sqrt{\tau}$ expression of the carbonation-front position obtained in $\mathrm{Pa}$ padakis et al. (AIChE J. 35:1639, 1989).

Keywords Fast reaction - Diffusion - Carbonation . Length scale separation · Water barrier · Matched asymptotics · Continuum mechanics

\section{Introduction}

The reaction-diffusion process studied in this paper concerns the following physico-chemical scenario: The reaction

$$
\begin{gathered}
\mathrm{CO}_{2}(\mathrm{~g} \rightarrow \mathrm{aq})+\mathrm{Ca}(\mathrm{OH})_{2}(\mathrm{~s} \rightarrow \mathrm{aq}) \\
\rightarrow \mathrm{H}_{2} \mathrm{O}+\mathrm{CaCO}_{3}(\mathrm{aq} \rightarrow \mathrm{s})
\end{gathered}
$$

takes place in all unsaturated porous cement-based materials exposed to air, where the reactants are assumed to be initially separated. The physicochemical process associated with (1) is called carbonation and can be summarized as follows: $\mathrm{CO}_{2}$ from the atmosphere penetrates the concrete material via the air parts of the pores and get absorbed in pore water. Once it is dissolved in pore water, aqueous $\mathrm{CO}_{2}$ moves to the places where aqueous $\mathrm{Ca}(\mathrm{OH})_{2}$ is available. The 
latter species comes from the pore matrix via dissolution $[16,17]$. The reactants meet and react cf. (1) to produce moisture $\left(\mathrm{H}_{2} \mathrm{O}\right)$ and carbonates $\left(\mathrm{CaCO}_{3}\right)$. The process is called carbonation and plays an important role in the service life of concrete-based structures. For more details on concrete carbonation and its relevance with respect to corrosion and durability issues of concrete-based structures, we refer the reader to the surveys by Kropp [13] and Chaussadent [6] as well as to the references cited therein.

In this paper, we rely on a conceptually simple isoline model to investigate a couple of asymptotic features of the carbonation process. The particularities of this model, which has been presented in [5] and is reformulated with minor modifications in Sect. 2, are the following: The reactant $\mathrm{CO}_{2}$ and the product $\mathrm{H}_{2} \mathrm{O}$ are allowed to diffuse, while the other reactant $\mathrm{Ca}(\mathrm{OH})_{2}$ is static. Furthermore, we assume that the total porosity of the concrete stays constant as well as that the effective diffusivities and reaction constant do not depend on humidity. Despite its apparent simplicity ${ }^{1}$, the model encompasses the main features of the carbonation process. In the sequel, we focus on the following issues:

(1) We study the asymptotic separation ${ }^{2}$ of length scales during carbonation arisen due to the strong competition between reaction and diffusion effects. We show that for sufficiently large times $\tau$ the width of the carbonated region is proportional to $\sqrt{\tau}$, while the width of the reaction front is proportional to $\tau^{\frac{p-1}{2(p+1)}}$ for carbonation-reaction rates with a power law structure like $k\left[\mathrm{CO}_{2}\right]^{p}[\mathrm{Ca}(\mathrm{OH})]_{2}^{q}$, where $k>0$ and $p, q>1$.

(2) We emphasize the occurrence of a water barrier in the reaction zone which hinders the penetration of $\mathrm{CO}_{2}$ via locally filling with water the air parts of the pores. This non-linear effect may be one of the causes why a purely linear extrapolation of accelerated carbonation test results to natural carbonation settings is not possible; For more discussions, see [19].

(3) We compare the asymptotic penetration law, which we obtain for the description of the position of the reaction front, against measured penetration depths extracted from [4]. The novelty consists in the fact that

\footnotetext{
${ }^{1}$ More elaborate models were proposed by several authors, see for instance [2, 12, 18, 24, 29, 31].

${ }^{2}$ The same issue was touched, for instance, in [3, 7, 14, 25, 33] from both theoretical and experimental viewpoints.
}

the factor multiplying $\sqrt{t}$ is now identified asymptotically by solving a non-linear system of ordinary differential equations, and hence, no fitting arguments are necessary to estimate its size. Our law may be viewed as another asymptotic alternative to the asymptotic $\sqrt{\tau}$ expression of the reaction-front position in [23]. In contrast to [23], we do not shrink the reaction layer to the corresponding free boundary. We rather prefer to keep the geometry of the layer as it is and estimate its width asymptotically taking into account the structure of the reaction kinetics.

\section{Comments on Refs. [3, 16, 21, 23, 30]}

Our motivation to apply formal asymptotic methods in order to tackle subjects like those stated in (1)-(3) stems from the pioneering approach to carbonation by Papadakis, Vayenas and Fardis in [23]. At the modeling level, we are influenced by the asymptotic investigation of $\mathrm{Ca}(\mathrm{OH})_{2}$ leaching in concrete done by Mainguy in his $\mathrm{PhD}$ thesis [16] and then applied in [17] by Mainguy and Coussy to the same problem. Similar ideas as in [16] were employed in the context of carbonation by Thiéry et al. in [30-32]. At the technical level, our approach is different (the working tool is the technique of matched asymptotics; see [1, 26], e.g., for details). The mathematical core of this contribution follows the methodology developed by Bazant and Stone in [3]. Nevertheless, the introduction of an additional partial differential equation to describe the diffusive behavior of the moisture produced by reaction (1) by means of which we point out asymptotically the presence of a water barrier, and also, our particular choice of scaling parameters used to nondimensionalize the model equations lead us away from the basic framework treated in [3]. However, since in the interior of the diffusion layer the equation of moisture formally decouples from those of the reactants, many of the working ideas employed in [3] become applicable to our setting. A mathematically new aspect here is the presence of a water barrier within the reaction layer. This paper is an essentially improved version of the preprint [21].

\section{Carbonation model}

We consider the carbonation model as proposed by Cahyadi and Uomoto in [5]. One of the particularities of their formulation is that the molecules of 
$\mathrm{Ca}(\mathrm{OH})_{2}$ and $\mathrm{CaCO}_{3}$ are supposed to be immobile (i.e. non-diffusing), while those of $\mathrm{CO}_{2}$ and of moisture may diffuse. Moreover, we consider that production and precipitation of $\mathrm{CaCO}_{3}$ are equal and do not significantly affect the remaining mechanisms of the reaction-diffusion problem in question.

Denote by $u, v$ and $w$ the dimensionless concentration of $\mathrm{CO}_{2}(\mathrm{aq}), \mathrm{Ca}(\mathrm{OH})_{2}(\mathrm{aq})$ and moisture produced by the reaction

$\mathrm{CO}_{2}+\mathrm{Ca}(\mathrm{OH})_{2} \rightarrow \mathrm{H}_{2} \mathrm{O}+\mathrm{CaCO}_{3}$.

Note that there are a few conceptual differences between (1) and (2). Particularly, we do not distinguish between the water and air phases. The main reason, which is behind such a treatment, is that both the transfer of $\mathrm{CO}_{2}$ from the air to water phase (and vice versa) and the dissolution of $\mathrm{Ca}(\mathrm{OH})_{2}$ from the solid matrix to water phase (and vice versa) may be assumed to be in local equilibrium, i.e. both production terms by Henry's law and by $\mathrm{Ca}(\mathrm{OH})_{2}$-dissolution vanish. Let $\Omega:=]-\infty,+\infty[$ and $S:=] 0, \infty[$ be the space and time domain of interest. Our choice of $\Omega$ underlines the fact that we do not account for boundary effects. Following [22, 23], the carbonation problem may be stated in dimensionless form as

$u_{, \tau}-\delta_{u} u_{, z z}=-\Phi^{2} f(u, v)$ equation for $\mathrm{CO}_{2}$

$\beta_{v} v_{, \tau}=-\Phi^{2} f(u, v)$ equation for $\mathrm{CaOH}_{2}$

$\beta_{w} w_{, \tau}-\delta_{w} w_{, z z}=+\Phi^{2} f(u, v)$

equation for $\mathrm{H}_{2} \mathrm{O}$

$$
\begin{gathered}
u(z, 0)=H(z), \quad v(z, 0)=H(-z) \quad \text { and } \\
w(z, 0)=0 \text { for all } z \in \Omega, \\
u(\infty, \tau)=1 \text { and } w(\infty, \tau)=\hat{w}, \\
u(-\infty, \tau)=0, w(-\infty, \tau)=\hat{w}
\end{gathered}
$$

for all $\tau \in S$. We are looking for left-traveling wave fronts of $\mathrm{CO}_{2}$ and $\mathrm{Ca}(\mathrm{OH})_{2}$ concentrations and for a pulse of humidity, see Fig. 1. These are solutions defined on the entire real line (see chapter 3 in [15], e.g.) and represent a mathematical ansatz of wave concentration profiles in the laboratory or in situ experiment.

The parameters $\delta_{u}, \delta_{w}, \beta_{v}, \beta_{w}$ and $\Phi^{2}$ are positive quantities which correspondingly represent the effective $\mathrm{CO}_{2}$ and humidity diffusivities, impact capacity

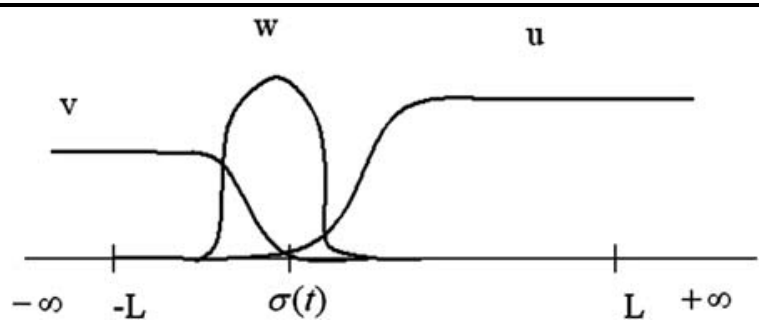

Fig. 1 Typical behavior of active concentrations $u, v$ and $w$

factors, and the Thiele modulus; see Sect. 8 for typical values. The term

$f(u, v):=u^{p} v^{q}$

denotes the production by carbonation reaction. The mass-balance equations (3)-(5) are coupled by means of $f$. In (6), $H(\cdot)$ is the Heaviside function. This is defined by

$H(\zeta)= \begin{cases}0, & \text { if } \zeta<0 \\ 1, & \text { if } \zeta \geq 0\end{cases}$

For simplicity, we assume in (7) that $\hat{w}=0$. Therefore, $w$ indicates the concentration of water produced by (1). Furthermore, since the species $v$ is not allowed to diffuse, it cannot leave its initial support, and hence, $v(x, t)=0$ for all $x>0$ and $t \geq 0$. The reactant $u$ diffuses into $-x$ direction toward the place hosting the static reactant $v$. Arguing as in [1,3], if an asymptotic similarity solution exist, then it must involve a moving front which diffuses into the material. See Sect. 3 and $[3,14,23]$, for discussions concerning why such a front is expected to exist. We denote by $\sigma(\tau)$ its position at the moment $\tau>0$. For fixing the ideas, let $\sigma(\tau)$ be the center of the reaction zone. We assume that $\frac{d \sigma(\tau)}{d \tau}<0$, where $\sigma(\tau)$ is given by

$\sigma(\tau):=-2 m \tau^{\alpha}, \quad \alpha \in \mathbb{R}, \tau \geq 0$.

The coefficient $m$ is a priori unknown. We expect $m$ to depend on the Thiele modulus $\Phi^{2}$, but the precise way in which this dependence holds still needs to be identified. We address the determination of $m$ in Sect. 7. The dependence of $m$ on $\Phi^{2}$ is explained for a particular case in Sect. 9. In the sequel, we omit to write down explicitly the dependence of $m$ on $\Phi^{2}$.

We use definition (10) of $\sigma(\tau)$ in order to distinguish between the three space domains of interest. Namely, we denote the carbonated zone (also 
called diffusion layer), the inter-phase carbonation layer (called also reaction layer) and the uncarbonated part, respectively, by

$\left.\Omega_{1}(\tau):=\right] \sigma(\tau)+\epsilon / 2, \infty[$,

$\Omega_{\epsilon}(\tau):=[\sigma(\tau)-\epsilon / 2, \sigma(\tau)+\epsilon / 2]$,

$\left.\Omega_{2}(\tau):=\right]-\infty, \sigma(\tau)-\epsilon / 2[$.

By (13), $\epsilon$ is the width of $\Omega_{\epsilon}(\tau)$. In this framework, $\Omega_{\epsilon}(\tau)$ some sort of a mushy region where $u, v$ and $w$ coexist. Since $\frac{d \sigma}{d \tau}<0$, the reaction front $\Omega_{\epsilon}(\tau)$ (and implicitly the production by reaction $f(u, v)$ ) moves towards the location of the source of $v$. The separation of reactants can be expressed as

$u(z, \tau)=0$ for all $z \in \Omega_{2}(\tau)$,

$v(z, \tau)=0 \quad$ for all $z \in \Omega_{1}(\tau)$.

Furthermore, we have

$w(z, \tau) \geq 0 \quad$ for all $z \in \Omega_{1}(\tau) \cup \Omega_{2}(\tau)$.

\section{Inner problem}

We consider as inner problem the scaling of the reaction layer $\Omega_{\epsilon}(\tau)$. We make use of the stretching variable

$\eta:=\frac{z-\sigma(\tau)}{\tau^{\beta}}=\frac{z+2 m \tau^{\alpha}}{\tau^{\beta}}$,

where we assume that the width $\epsilon$ of $\Omega_{\epsilon}(\tau)$ is proportional to $\tau^{\beta}$ for some $\beta \geq 0$. By (17), we deduce

$\eta_{, z}=\tau^{-\beta} \quad$ and $\quad \eta_{, \tau}=-\beta \tau^{-1} \eta+2 m \alpha \tau^{\alpha-\beta-1}$.

In the vicinity of $\sigma(\tau)$, we allow the concentrations $u$ and $w$ to vary like

$$
\begin{gathered}
U(\eta, \tau):=\tau^{\gamma} u(z, \tau), \quad V(\eta, \tau)=v(x, t) \quad \text { and } \\
W(\eta, \tau)=\tau^{v} w(z, \tau)
\end{gathered}
$$

with $\gamma>0$ and $v>0$. We refer the reader to [3], for a discussion of the non-relevance of the cases $\gamma=0$ and $v=0$.

Owing to (19), it is necessary to replace $f(u, v)$ in (8) by $f(U, V)=\tau^{-\delta} U^{p} V^{q}$, with $\delta:=\gamma p$.
By (17), (18), (19) and the chain rule, the massbalance equations (3), (4) and (5) become

$$
\begin{gathered}
\tau^{\gamma(p-1)} U_{, \tau}-\tau^{\gamma(p-1)-1}\left(\beta \eta U_{, \eta}+\gamma U\right) \\
+2 m \alpha \tau^{\alpha-\beta+\gamma(p-1)-1} U_{, \eta} \\
=\tau^{\gamma(p-1)-2 \beta} \delta_{u} U_{, \eta \eta}-\Phi^{2} U^{p} V^{q}, \\
\tau^{\gamma p} V_{, \tau}-\tau^{\gamma p-1} \beta \eta V_{, \eta}+2 m \alpha \tau^{\alpha-\beta+\gamma p-1} V_{, \eta} \\
=-\frac{\Phi^{2}}{\beta_{v}} U^{p} V^{q}, \\
\tau^{\gamma p-v} W_{, \tau}-\tau^{\gamma p-\nu-1}\left(\beta \eta W_{, \eta}+v W^{2}\right) \\
+2 m \alpha \tau^{\alpha-\beta+\gamma p-\nu-1} W_{, \eta} \\
=\tau^{p \gamma-\nu-2 \beta} \frac{\delta_{w}}{\beta_{w}} W_{, \eta \eta}+\frac{\Phi^{2}}{\beta_{w}} U^{p} V^{q} .
\end{gathered}
$$

We aim at finding asymptotically invariant (similarity) solutions which correspond to the following limiting behavior with suitable $\hat{U}, \hat{V}$ and $\hat{W}$ :

$U(\eta, \tau) \rightarrow \hat{U}(\eta), \quad V(\eta, \tau) \rightarrow \hat{V}(\eta)$,

$W(\eta, \tau) \rightarrow \hat{W}(\eta), \quad U_{, \eta}(\eta, \tau) \rightarrow \hat{U}_{, \eta}(\eta)$,

$V_{, \eta}(\eta, \tau) \rightarrow \hat{V}_{, \eta}(\eta), \quad W_{, \eta}(\eta, \tau) \rightarrow \hat{W}_{, \eta}(\eta)$,

$U_{, \eta \eta}(\eta, \tau) \rightarrow \hat{U}_{, \eta \eta}(\eta), \quad W_{, \eta \eta}(\eta, \tau) \rightarrow \hat{W}_{, \eta \eta}(\eta)$

as $\tau \rightarrow \infty$ and $-\infty<\eta<\infty$. We make use of the same arguments as in [3] to require the presence of a dominant balance (see also chapter 5 in [11], e.g.), which compensates the effect of the nonlinear reaction $U^{p} V^{q}$. The first step needed in order to obtain the time-invariance of $U, V$ and $W$ is to require the smallness of the terms $U_{, \tau}, V_{, \tau}$ and $W_{, \tau}$ relatively to the production by reaction. In other words, we assume the pseudo-steady state approximation for $U, V$ and $W$, i.e.

$$
\begin{gathered}
\lim _{\tau \rightarrow \infty} \tau^{\gamma(p-1)} U_{, \tau}=\lim _{\tau \rightarrow \infty} \tau^{\gamma p} V_{, \tau} \\
=\lim _{\tau \rightarrow \infty} \tau^{\gamma p-\nu} W_{, \tau}=0 .
\end{gathered}
$$

The dominant balance situations for $U, V$, and $W$ suggest the study of the following cases:

(U1) $\gamma(p-1)-2 \beta=0, \alpha-\beta+\gamma(p-1)-1 \leq 0$, $\gamma(p-1)-1 \leq 0$, 
(U2) $\gamma(p-1)-2 \beta \leq 0, \alpha-\beta+\gamma(p-1)-1=0$, $\gamma(p-1)-1 \leq 0$

(U3) $\gamma(p-1)-2 \beta \leq 0, \alpha-\beta+\gamma(p-1)-1 \leq 0$, $\gamma(p-1)-1=0$,

(V1) $\gamma p-1=0, \alpha-\beta+\gamma p-1 \leq 0$,

(V2) $\gamma p-1 \leq 0, \alpha-\beta+\gamma p-1=0$,

and

(W1) $p \gamma-v-2 \beta=0, \alpha-\beta+\gamma p-v-1 \leq 0$, $\gamma p-v-1 \leq 0$

(W2) $p \gamma-v-2 \beta \leq 0, \alpha-\beta+\gamma p-v-1=0$, $\gamma p-v-1 \leq 0$

(W3) $p \gamma-v-2 \beta \leq 0, \alpha-\beta+\gamma p-v-1 \leq 0$, $\gamma p-v-1=0$.

We proceed as in [3] and find out the traveling wave cases (U2, V2, W1) and (U2, V2, W2) and the diffusing front case (U1, V2, W1). We drop those configurations which are not logically consistent ${ }^{3}$, see the Appendix for details. We are not interested in any traveling wave, but only on those that are bounded and approach constant, equilibrium states at $z= \pm \infty$. These special types of traveling wave solutions are called wave front (or diffusing front) solutions. On this way, we are only left with the diffusing front case (U1, V2, W1). Therefore, we are forced to take into account two regions with different scale invariance: one for the diffusion layer, and another one for the reaction layer. In this case the front advances sub-linearly in time, i.e. like $\tau^{\alpha}$ with $0<\alpha<1$. Note that such a sub-linear behavior of the front has been mathematically shown for two moving-boundary formulations of the concrete carbonation problem in [22].

\section{Outer problem}

In this section, we deal with the case of the infinitely thin carbonation front (layer). Namely, we only consider the case when Thiele modulus $\Phi^{2}$ satisfies $\infty>\Phi^{2} \gg 1$; see also appendix A in [22] or [23]. It is well known that in the carbonation process the reaction is faster than the transport. Therefore, we expect that the width $\epsilon>0$ of the reaction layer $\Omega_{\epsilon}(\tau)$

${ }^{3}$ For the case (U1, V2, W1), we have $v=\gamma, 0<\gamma \leq \frac{1}{p}$, $\beta=\frac{\gamma}{2}(p-1), \alpha \leq 1-\frac{\gamma}{2}(p-1)$. One can show that the traveling-wave ansatz is not satisfactory for the reactiondiffusion model in question. We do not repeat the arguments here and refer the reader to [3] (Equations (22a)-(23)). is relatively small compared to the width of the diffusion layer $\Omega_{1}(\tau)$. Such setting occurs when in the reaction-diffusion process the characteristic time scale of reaction is much smaller than that of diffusion. The singular case $\Phi^{2}=\infty$ will be considered elsewhere.

We consider as outer problem the scaling of the diffusion layer $\Omega_{1}(\tau)$. To scale this region, we introduce another similarity variable which reads

$\zeta:=\frac{2 m \tau^{\alpha}+z}{2 \tau^{\rho}}$ for all $\zeta>0, \zeta \geq \sigma$.

In (25), we have $\rho>0$. The similarity variables $\eta$ cf. (17) and $\zeta$ cf. (25) are linked through

$\eta=2 \tau^{\rho-\beta} \zeta$.

If $\eta \rightarrow \infty$ and $\zeta \rightarrow 0$ with $\zeta>0$, then the carbonated zone needs to connect with the reaction layer. Let us introduce some new functions

$\mathcal{U}(\zeta, \tau):=u(z, \tau), \mathcal{V}(\zeta, \tau):=v(z, \tau)$ and

$$
\mathcal{W}(\zeta, \tau):=w(z, \tau)
$$

As in Sect. 3, we omit to write the dependence of $m$ on $\Phi^{2}$. (25) yields

$\zeta_{, z}=\frac{1}{2 \tau^{\rho}} \quad$ and $\quad \zeta_{, \tau}=m \alpha \tau^{\alpha-\rho-1}-\zeta \rho \tau^{-1}$.

The mass-balance equations (3)-(5) become

$$
\begin{gathered}
\tau^{2 \rho} \mathcal{U}_{, \tau}+m \alpha \tau^{\alpha+\rho-1} \mathcal{U}_{, \zeta}-\zeta \rho \tau^{2 \rho-1} \mathcal{U}_{, \zeta} \\
=\frac{1}{4} \delta_{u} \mathcal{U}_{\zeta \zeta}-\Phi^{2} \tau^{2 \rho} \mathcal{U}^{p} \mathcal{V}^{q}
\end{gathered}
$$

$$
\begin{aligned}
& \tau^{2 \rho} \mathcal{V}_{, \tau}+m \alpha \tau^{\alpha+\rho-1} \mathcal{V}_{, \zeta}-\zeta \rho \tau^{2 \rho-1} \mathcal{V}_{, \zeta} \\
& =-\frac{\Phi^{2}}{\beta_{v}} \tau^{2 \rho} \mathcal{U}^{p} \mathcal{V}^{q},
\end{aligned}
$$

and

$$
\begin{gathered}
\tau^{2 \rho} \mathcal{W}_{, \tau}+m \alpha \tau^{\alpha+\rho-1} \mathcal{W}_{, \zeta}-\zeta \rho \tau^{2 \rho-1} \mathcal{W}_{, \zeta} \\
=\frac{1}{4} \frac{\delta_{u}}{\beta_{w}} \mathcal{W}_{\zeta \zeta}-\frac{\Phi^{2}}{\beta_{w}} \tau^{2 \rho} \mathcal{U}^{p} \mathcal{V}^{q} .
\end{gathered}
$$

We look for capturing the following asymptotic similarity behavior

$\mathcal{U}(\zeta, \tau) \rightarrow \hat{\mathcal{U}}(\zeta), \quad \mathcal{V}(\zeta, \tau) \rightarrow \hat{\mathcal{V}}(\zeta)$ 
$\mathcal{W}(\zeta, \tau) \rightarrow \hat{\mathcal{W}}(\zeta), \quad \mathcal{U}_{, \zeta}(\zeta, \tau) \rightarrow \hat{\mathcal{U}}_{, \zeta}(\zeta)$,

$\mathcal{V}_{, \zeta}(\zeta, \tau) \rightarrow \hat{\mathcal{V}}_{, \zeta}(\zeta), \quad \mathcal{W}_{, \zeta}(\zeta, \tau) \rightarrow \hat{\mathcal{W}}_{, \zeta}(\zeta)$,

$\mathcal{U}_{, \zeta \zeta}(\zeta, \tau) \rightarrow \hat{\mathcal{U}}_{, \zeta \zeta}(\zeta), \quad \mathcal{W}_{, \zeta \zeta}(\zeta, \tau) \rightarrow \hat{\mathcal{W}}_{, \zeta \zeta}(\zeta)$,

as $\tau \rightarrow \infty$ and $\zeta>0$. In order to realize this, we firstly assume that the time derivatives of $\mathcal{U}, \mathcal{V}$ and $\mathcal{W}$ can be neglected when compared to diffusion terms

$\lim _{\tau \rightarrow \infty} \tau^{2 \rho} \mathcal{U}, \tau(\zeta, \tau)=\lim _{\tau \rightarrow \infty} \tau^{2 \rho} \mathcal{W}, \tau(\zeta, \tau)=0$

for all $\zeta>0$.

A scaling, which is different from that one used for the carbonation layer, can be acquired if the production by reaction does not enter the dominant balance. Thus, we assume that

$\lim _{\tau \rightarrow \infty} \tau^{2 \rho} \Phi^{2} \mathcal{U}^{p}(\zeta, \tau) \mathcal{V}^{q}(\zeta, \tau)=0$ for all $\zeta>0$.

The investigation of the dominant balance suggests the following possible cases:

$\alpha+\rho-1=0, \quad 2 \rho-1 \leq 0$

$\alpha+\rho-1 \leq 0, \quad 2 \rho-1=0$.

The situation corresponding to $\alpha+\rho-1 \leq 0$ and $2 \rho-1=0$ represents the case of the diffusing frontlike solution, the width of the carbonated region being then proportional to $\sqrt{\tau}$. The other situation is eliminated as in [3].

\section{Matching of the inner and outer approximations}

The limit $\eta \rightarrow \infty$ of the inner approximation has to match the limit $\zeta \rightarrow 0, \zeta>0$ of the outer approximation. Since finally we want to obtain an asymptotic matching, it is essential to pass to the limit $\tau \rightarrow \infty$ before the matching is realized. The major care is to propose those matching conditions which conserve the mass when passing from the diffusion layer to the reaction layer.

The matching of $v$ is

$\lim _{\eta \rightarrow \infty} \hat{V}(\eta)=0=\lim _{\zeta \rightarrow 0^{+}} \hat{\mathcal{V}}(\zeta)$.

To match the outer and inner limits of $u$ and $w$, some care is needed to formulate the connecting conditions in the intermediate zone. This intermediate region lies between the reaction and diffusion layer. Note firstly that since $\gamma>0$ the concentration profiles of $u$ and $w$ decay to zero as $\tau \rightarrow \infty$ and $|\eta|<\infty$. The decay happens like $u(z, \tau)=\mathcal{O}\left(\tau^{-\gamma}\right)$ and $w(z, \tau)=\mathcal{O}\left(\tau^{-\nu}\right)$. We proceed as in [3] and match the linear terms in the Taylor expansion of $u$ and $w$ in the intermediate region. Notice that

$u_{, z}(z, \tau)=\frac{\hat{\mathcal{U}}^{\prime}(\zeta)}{2 \tau^{\rho}}, \quad w_{, z}(z, \tau)=\frac{\hat{\mathcal{W}}^{\prime}(\zeta)}{2 \tau^{\rho}}$,

as $\tau \rightarrow \infty$ and $0<\zeta<\infty$ is fixed, and

$u_{, z}(z, \tau)=\frac{1}{\tau^{\beta+\gamma}} \hat{U}^{\prime}(\eta)$,

$w_{, z}(z, \tau)=\frac{1}{\tau^{\beta+\nu}} \hat{W}^{\prime}(\eta)$,

as $\tau \rightarrow \infty$ and $|\eta|<\infty$ is fixed. Equations (34) and (35) suggest the matching of the exponents

$\beta+\gamma=\rho, \quad \beta+v=\rho$

and also

$\hat{U}^{\prime}(\infty)=\frac{1}{2} \hat{\mathcal{U}}^{\prime}(0), \quad \hat{W}^{\prime}(\infty)=\frac{1}{2} \hat{\mathcal{W}}^{\prime}(0)$.

The values of $\hat{U}^{\prime}(\infty)$ and $W^{\prime}(\infty)$ will be calculated in Sect. 6. Finally, we conclude this section by giving the asymptotic scaling we were looking for:

$\beta=\frac{p-1}{2(p+1)}, \quad \gamma=\frac{1}{p+1}$,

$\rho=\alpha=\frac{1}{2}, \quad \delta=\frac{p}{p+1}=p \gamma$,

and recall the relation $v=\gamma$. It is worth mentioning that the value of $\gamma$ in (38) is obtained combining

$\beta+\gamma=\rho \quad$ and $\quad \beta=\frac{\gamma}{2}(p-1)$.

\section{Calculation of $\hat{U}^{\prime}(\infty)$ and $\hat{W}^{\prime}(\infty)$}

The concentration profiles of $\hat{U}$ and $\hat{W}$ can be calculated in the diffusion layer. Herein, we have

$2(m-\eta) \hat{U}^{\prime}=\delta_{u} \hat{U}^{\prime \prime}$

$\hat{U}(0)=0, \quad \hat{U}(\infty)=1$ 
and hence,

$-2\left(\frac{\eta}{\delta_{u}}-\frac{m}{\delta_{u}}\right) \hat{U}^{\prime}=\hat{U}^{\prime \prime}$

$\hat{U}(0)=0, \quad \hat{U}(\infty)=1$.

The exact solution of (39)-(40) is

$\hat{U}(\eta)=\frac{\operatorname{erf}\left(\frac{\eta}{\delta_{u}}-\frac{m}{\delta_{u}}\right)+\operatorname{erf}\left(\frac{m}{\delta_{u}}\right)}{1+\operatorname{erf}\left(\frac{\mathrm{m}}{\delta_{\mathrm{u}}}\right)}$,

where the error function erf is defined by

$\operatorname{erf}(\lambda)=\frac{2}{\sqrt{\pi}} \int_{0}^{\lambda} \exp \left(-r^{2}\right) d r$

for all $\lambda \geq 0$. Similarly, we obtain

$\hat{W}(\eta)=0$ for all $\eta \geq 0$.

Denoting

$M_{u}:=\frac{m}{\delta_{u}} \quad$ and $\quad \alpha:=\frac{\eta}{\delta_{u}}$,

we can write

$$
\begin{aligned}
\frac{U^{\prime}(0)}{2}=\lim _{\eta \rightarrow 0} \frac{\hat{U}^{\prime}(\eta)}{2}= & \lim _{\eta \rightarrow 0} \frac{1}{\sqrt{\pi}\left(1+\operatorname{erf}\left(M_{u}\right)\right)} \\
& \times \exp \left(-\left(\alpha-M_{u}\right)^{2}\right)
\end{aligned}
$$

$\frac{W^{\prime}(0)}{2}=\lim _{\eta \rightarrow 0} \frac{\hat{W}^{\prime}(\eta)}{2}=0$.

The matching conditions allow us to define $U^{*}$ and $W^{*}$ via

$U^{*}=\hat{U}^{\prime}(\infty)=\frac{U^{\prime}(0)}{2}$,

$W^{*}=\hat{W}^{\prime}(\infty)=\frac{W^{\prime}(0)}{2}$.

\section{Identification of $m$ in (10)}

Let us consider the following system of ordinary differential equations which hold in the reaction front:

$\delta_{u} \hat{\mathcal{U}}^{\prime \prime}-\Phi^{2} \hat{\mathcal{U}}^{p} \hat{\mathcal{V}}^{q}=0$

$m \hat{\mathcal{V}}^{\prime}-\frac{\Phi^{2}}{\beta_{v}} \hat{\mathcal{U}}^{p} \hat{\mathcal{V}}^{q}=0$, $\delta_{w} \hat{\mathcal{W}}^{\prime \prime}+\frac{\Phi^{2}}{\beta_{w}} \hat{\mathcal{U}}^{p} \hat{\mathcal{V}}^{q}=0$.

The associated boundary conditions are

$\hat{\mathcal{U}}(-\infty)=0 \quad \hat{\mathcal{U}}^{\prime}(\infty)=U^{*}$,

$\hat{\mathcal{V}}(-\infty)=1 \quad \hat{\mathcal{V}}(\infty)=0$,

$\hat{\mathcal{W}}(-\infty)=0 \quad \hat{\mathcal{W}}^{\prime}(\infty)=W^{*}$,

where the boundary values $U^{*}$ and $W^{*}$ are given by (46) and (47). It is important to observe that we need all 6 boundary conditions listed in (51)-(53) in order to find the solution $\hat{\mathcal{U}}, \hat{\mathcal{V}}$ and $\hat{\mathcal{W}}$ of the system (48)-(50), and simultaneously, to identify the parameter $m$. Note that $\hat{\mathcal{U}}, \hat{\mathcal{V}}, \hat{\mathcal{W}}$ and $m$ depend on the choice of $\delta_{w}, \beta_{v}, \beta_{w}$ and $\Phi^{2}$. Combining ${ }^{4}$ equations (51) and (52) and then integrating the result firstly from $\zeta$ up to $\infty$, and afterwards, integrating the result mentioned above from $-\infty$ up to $\zeta$ yields

$\delta_{u} U^{*}-\delta_{u} \hat{\mathcal{U}}^{\prime}(\zeta)-\beta_{v} m \hat{\mathcal{V}}(\zeta)=0$ and

$-\beta_{v} m+\beta_{v} m \hat{\mathcal{V}}(\zeta)+\delta_{u} \hat{\mathcal{U}}^{\prime}(\zeta)=0$,

for all $\zeta \geq 0$, and hence,

$m=\frac{\delta_{u} U^{*}(m)}{\beta_{v}}$.

Equation (55) has a uniquely determined solution $m>0$, see Fig. 2. This type of reasoning is standard if

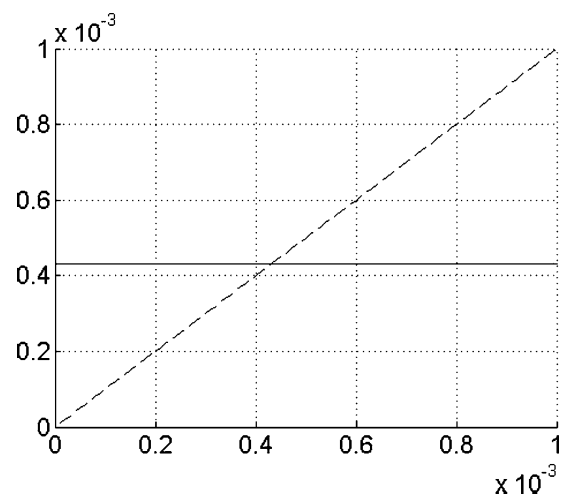

Fig. 2 Calculation of $m$ for the standard set of parameters used in Sect. 8. The dotted line is the 1.h.s. of (55), while the solid line is the corresponding r.h.s.

${ }^{4}$ See also [15] pp. 78-79, e.g. 
we think of calculating wave speeds. With the notation

$\Theta:[0, \infty[\rightarrow \mathbb{R}$ defined by

$\Theta(\chi):=\frac{1}{\beta_{v}}\left[\frac{e^{-\chi^{2}}}{\sqrt{\pi}(1+\operatorname{erf}(\chi))}\right]$,

we get $U^{*}(\chi)=\beta_{v} \Theta(\chi)$ for all $\chi \geq 0$. It is straightforward to eliminate $\hat{\mathcal{V}}$ to obtain a single equation in terms of $\hat{\mathcal{U}}$. By (48), (49) and (54), we obtain

$\hat{\mathcal{U}}^{\prime}(\zeta)=U^{*}-\frac{\beta_{v} m}{\delta_{u}} \hat{\mathcal{V}}(\zeta)=U^{*}(1-\hat{\mathcal{V}}(\zeta))$

for $\zeta \geq 0$

and hence,

$\hat{\mathcal{V}}(\zeta)=1-\frac{\hat{\mathcal{U}}(\zeta)}{U^{*}}, \quad \zeta \geq 0$

Thus (48) becomes

$\delta_{u} \hat{\mathcal{U}}^{\prime \prime}-\Phi^{2} \hat{\mathcal{U}}^{p}\left(1-\frac{\hat{\mathcal{U}}^{\prime}}{U^{*}}\right)^{q}=0$.

The boundary conditions (51) are associated to the non-linear equation (59). Questions concerning the existence and uniqueness of the reaction front scaling function $\hat{\mathcal{U}}$, which satisfies (59) and (51), were addressed in [3], section 3.6. Once $\hat{\mathcal{U}}$ is known, the system (48)-(53) decouples and the remaining equations are then easy to integrate.

\section{Simulation results}

We turn our attention to the carbonation setting described in [4]. We perform the calculations with respect to the space domain $\left.\Omega_{L}:=\right]-L, L[$ and the time interval $\left.S_{T}:=\right] 0, T[$. In this section, we set $L=1 \mathrm{~cm}$, $\epsilon=\frac{1}{7} \mathrm{~cm}$ and $T=18$ years. Note that $T$ represents the final time of the carbonation process in Bunte's experiment (cf. [4]) and $L>\epsilon$. The couple $\left(\Omega_{L}, S_{T}\right)$ is used for the numerical approximation of the infinitely large intervals $\Omega$ and $S$. Further, let us denote by $D_{A}$ the effective diffusivity of species $A$, by $\lambda_{A}$ the Dirichlet boundary datum which we assume to be compatible with the corresponding initial datum. Our candidates for $A$ are the reactants and products involved in (1). By $\eta_{\max }$ we denote the maximum value of the reaction rate.
Herein, the following material parameters are used: $D_{\mathrm{CO}_{2}}=3.5 \mathrm{~cm}^{2} /$ day, $\quad D_{\mathrm{H}_{2} \mathrm{O}}=1 \mathrm{~cm}^{2} /$ day, $\lambda_{\mathrm{CO}_{2}}=58.9286 * 10^{-6} \mathrm{~g} / \mathrm{cm}^{3}, \lambda_{\mathrm{Ca}(\mathrm{OH})_{2}}=0.077 \mathrm{~g} / \mathrm{cm}^{3}$, $\lambda_{\mathrm{H}_{2} \mathrm{O}}=1 \mathrm{~g} / \mathrm{cm}^{3}, \quad \eta_{\max }=k \lambda_{\mathrm{CO}_{2}}^{p} \lambda_{\mathrm{Ca}(\mathrm{OH})_{2}}^{q}, \quad$ where $k=150 \quad\left[1 /\right.$ day* $\left.\left(\mathrm{g} / \mathrm{cm}^{3}\right)^{1-p-q}\right]$. Moreover, we fix $p=2$ and $q=1$ but other choices are possible as well. By (38), we have $\beta(p)=\frac{p-1}{2(p+1)}$, and hence, we expect results which are asymptotically insensitive with respect to the selection of the partial-reaction order $q$. Since we are in the fast reaction vs. slow diffusion case, we expect that asymptotically the overall influence of the exponents $p$ and $q$ on the concentration profiles and position of the reaction front is negligi$b^{5} e^{5}$. These parameters define the following dimensionless numbers:

$\beta_{u}:=1, \quad \beta_{v}:=\frac{\lambda_{\mathrm{Ca}(\mathrm{OH})_{2}}}{\lambda_{\mathrm{CO}_{2}}}, \quad \beta_{w}:=\frac{\lambda_{\mathrm{H}_{2} \mathrm{O}}}{\lambda_{\mathrm{CO}_{2}}}$,

and

$\delta_{u}:=1, \quad \delta_{v}:=\frac{D_{\mathrm{Ca}(\mathrm{OH})_{2}}}{D_{\mathrm{CO}_{2}}}, \quad \delta_{w}:=\frac{D_{\mathrm{H}_{2} \mathrm{O}}}{D_{\mathrm{CO}_{2}}}$.

For simulations, we choose $\epsilon=10^{-2}$ to scale the length variable and the characteristic time of $\mathrm{CO}_{2}$ diffusion to scale the time variable. With these notations, the Thiele modulus reads

$\Phi^{2}:=\frac{\eta_{\max } \epsilon^{2}}{D_{\mathrm{CO}_{2}} \lambda \mathrm{CO}_{2}}$.

Thorough discussions of the role of these dimensionless numbers are given in [23] and in appendix A of [22]. Our focus is on calculating the asymptotic profiles of the involved active concentrations and of the asymptotic reaction front position. We begin with the next elementary observation: After a sufficient large time, the behavior of $u$ within the diffusion layer is described via

$u_{\tau}-\delta_{u} u_{z z}=0 \quad$ in $\mathbb{R}, \tau>0$,

$u(z, 0)=H(z), \quad u(-\infty, \tau)=0, \quad u(\infty, \tau)=1$.

${ }^{5}$ For $p=2$, (38) shows that $\epsilon$ is of order of $\mathcal{O}\left(\tau^{\frac{1}{6}}\right)$ which is negligible compared to the width of $\Omega_{L}$. Following the same arguments, if $p=1.5$, then $\epsilon \sim \mathcal{O}\left(\tau^{\frac{1}{10}}\right)$. Interestingly, if $p=1$, then $\epsilon$ and $\tau$ appears to be asymptotically independent, while $\beta(p) \rightarrow \frac{1}{2}$ as $p \rightarrow \infty$. 
The system (63)-(64) admits the exact similarity solution

$u(z, \tau)=\frac{1}{2}\left(1+\operatorname{erf}\left(\frac{z}{2 \sqrt{\beta_{u} \tau}}\right)\right)$.

In terms of $(\zeta, \tau)$ variables, the latter equation reads

$u(\zeta, \tau)=\frac{1}{2}\left[1+\operatorname{erf}\left(\frac{1}{\sqrt{\beta_{u}}}(\zeta-m)\right)\right]$.

Simultaneously, by the maximum principle the profile of $w$ vanishes in the same region. The profile of $u$ cf. (65) is shown in Fig. 3. It shows the tendency of $\mathrm{CO}_{2}$ to decay near the reaction layer.

In the sequel, we want to obtain the asymptotic profiles of concentrations in the reaction layer. In order to obtain them, we need to solve the non-linear system of differential equations (48)-(53), which is described in Sect. 7. It is worth mentioning that in [23] the effect of the reaction layer on the whole process was neglected. The approximation of the solution to the boundaryvalue problem (48), (51) and (58) is obtained by means of the code bvp $4 \mathrm{c}$, which is available in MATLAB, using default tolerances. To this end, the problem has to be transformed to first order form. The routine is based on both the collocation method and continuation argument, and is illustrated in [27] (chapter 3.2), e.g. the numerical approximation of the reactant profiles in Fig. 4 is done within the reaction front. Firstly, we approximate the value of $m$ for a given set of parameters by solving (55) in MATLAB by means of the routine fminsearch. The profile of $u$ is depicted in Fig. 4(a), while that of $v$ is shown in Fig. 4(b). In Fig. 5, we note a localized production of water which may be interpreted as a barrier. By barrier we simply mean that the water may locally fill the air part of some

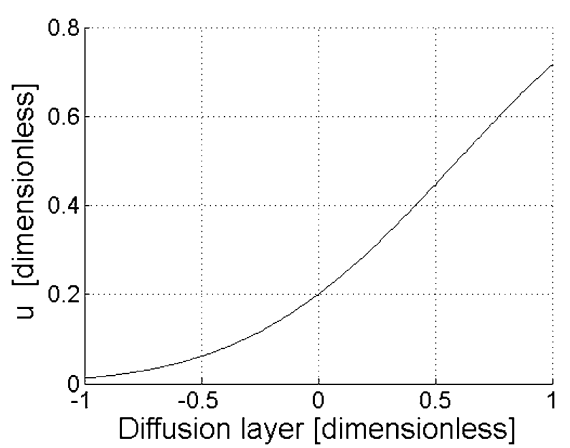

Fig. 3 Profile of $\mathrm{CO}_{2}$ vs. space within the diffusion layer pores. On this way, the water barrier impedes the penetration of gaseous $\mathrm{CO}_{2}$ and may lead to a slow down of the process or even to its stopping.

Asymptotically, the reaction front diffuses into the material with the speed

$\sigma^{\prime}(\tau):=-\frac{m}{\sqrt{2 \tau}}$,

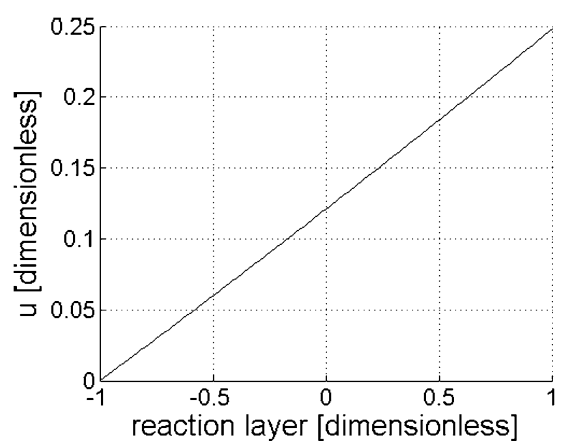

(a)

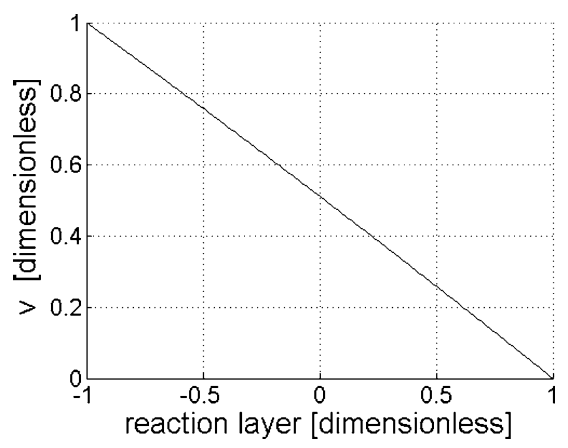

(b)

Fig. 4 (a) $+\left(\right.$ b) $\mathrm{CO}_{2}$ and $\mathrm{Ca}(\mathrm{OH})_{2}$ profiles vs. space. The reaction front propagates from right to left

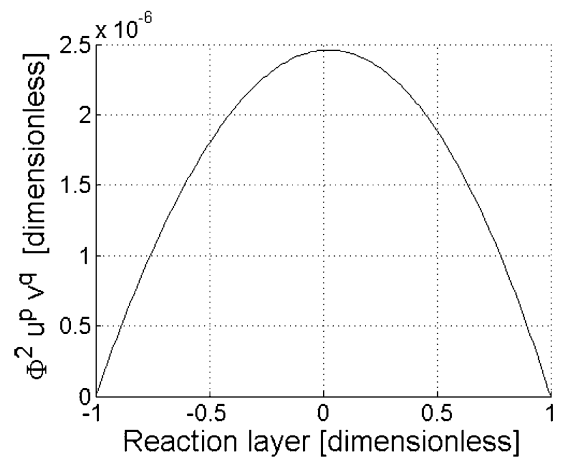

Fig. 5 Plot of the reaction rate $\Phi^{2} \hat{\mathcal{U}}^{p} \hat{\mathcal{V}}^{q}$ vs. space within the reaction layer. It points out a local production of water 


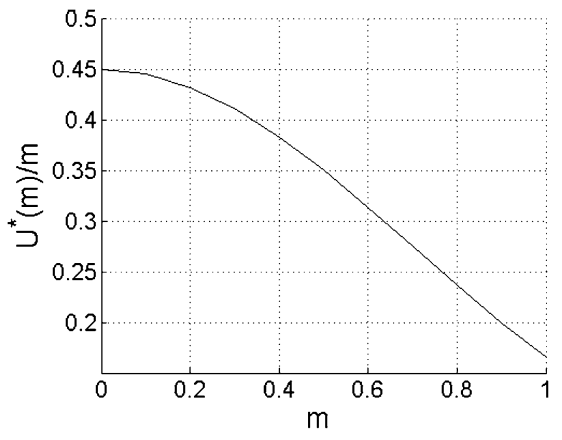

Fig. 6 Behavior of $\frac{U^{*}(m)}{m}$ vs. $m$

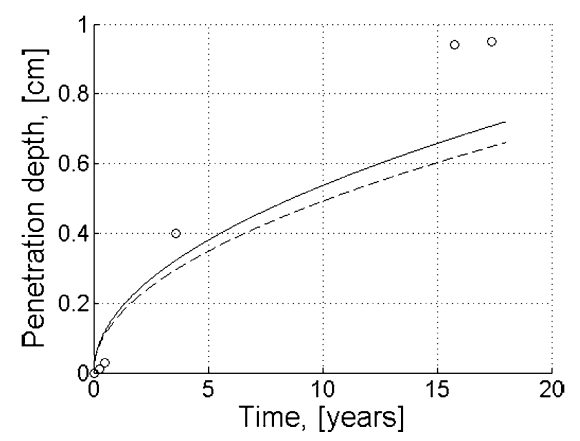

(a)

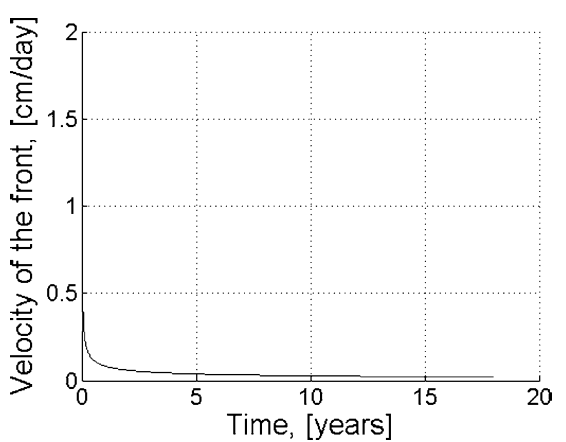

(b)

Fig. 7 (a) The solid line shows the interface position cf. (10) vs. time. The dashed line show the interface position cf. (67) vs. time. The points "o" denote the measured penetration depths from [4]. (b) Plot of the velocity (66) of the front

see Fig. 7(a) for a plot of the (asymptotical) interface position with respect to time compared with the experimental penetration depth measured by Bunte [4]. In Fig. 7(a) we also compare the position of the reaction front given by (10) with that respecting the law

$\sigma(\tau)=-\sqrt{\frac{2 \lambda_{c} D_{\mathrm{CO}_{2}} \tau}{\lambda_{h}}}$, which is basically the law proposed by Papadakis et al. [23] applied to this reaction-diffusion scenario. The results are comparable. For the chosen parameter set, our approach seems to be better. ${ }^{6}$ The velocity of the front is plotted in Fig. 7(b).

\section{Discussion}

There is no explicit dependence ${ }^{7}$ of $m$ defined cf. (55) on important material parameters like the curing time, degree of hydration, water-to-cement ratio, chemistry of the concrete and so on. Therefore, the criticism on the penetration law proposed in [23], which was addressed among others by Chaussadent in [6], can be repeated here as well. There are indeed arguments pro et contra. We do not dwell with them here, but emphasize that the main merit of such an asymptotic approach (application of the methodology developed in [3]) is that it eliminates any fitting argument when determining the position of the reaction front. In our case we obtain the unknown parameter $m$ as solution of a system of differential equations coupled with an algebraic one. The information concerning the chemistry of the concrete sample and the boundary conditions is comprised in the scaling parameters. Therefore, the asymptotic penetration law only depends on the choice of scaling parameters and that of $m$. Our asymptotic penetration depths are close to those obtained by $\mathrm{Pa}$ padakis et al. [23]. Note that, at this moment, it is not quite clear whether we overestimate or underestimate their results.

Exploiting (55), we note that $\frac{U^{*}(m)}{m}=\mathcal{O}\left(\frac{1}{\Phi^{2}}\right)$. This can be interpreted in the following way: For increasing $m$, the fraction $\frac{U *(m)}{m}$ decreases, see Fig. 6. This also corresponds to increasing Thiele modulus $\Phi^{2}$ and finally leads cf. (10) to higher penetration depths. Simple dimensional investigation shows that the proportionality constant $c$ from

$\frac{U^{*}(m)}{m}=c \Phi^{2}$

depends on the effective diffusion coefficient of $\mathrm{CO}_{2}$ in air, maximum reaction rate, choice of length scale

\footnotetext{
${ }^{6}$ This is not yet concluding. More qualitative investigations are needed.

${ }^{7}$ However, the scaling parameters connect $m$ with the initial and boundary data.
} 
and initial concentration of $\mathrm{Ca}(\mathrm{OH})_{2}$ in concrete. If $m$ is known, then by (55) the constant $c$ can be exactly computed. In this case, we have

$c=\frac{D_{\mathrm{CO}_{2}} \lambda \mathrm{Ca}(\mathrm{OH})_{2}}{\eta_{\max } \epsilon^{2}}$,

where $\epsilon$ represents the characteristic length scale of interest. Furthermore, for certain values of $m$, we notice the linear behavior $\Phi^{2}(m) \approx \xi m$, where the approximate proportionality factor $\xi$ can be calculated from Fig. 6 with the routine diff of MATLAB.

If we change the set of scaling parameters, then the main output (i.e. penetration depth vs. time plot) alters. More dimensional investigations as well as numerical simulations are needed to identify the effect of each of the dimensionless parameters and dimensional length and time scales on the penetration depths.

At least two complementary research directions emerge from the preliminary character of this study. From one hand, the matched asymptotic analysis can be extended to tackle rather complex fast reaction scenarios in which several reactive species can diffuse and admit various structures of reaction kinetics (e.g. a humidity-dependent rate like $\left.f(u, v, w)=w u^{p} v^{q}\right)$. Typically, tedious calculations become unavoidable. The paper [3] and this note provide a basis for investigations in this direction. Furthermore, the asymptotic results may be combined with numerics. On the other hand, the matched asymptotic analysis offers some theoretical hints about the large-time behavior of concentration profiles and penetration front position, at least in the case when the front is thin (but does not shrink to a sharp interface). There are essential differences between the asymptotic behavior of moving thin fronts and sharp interfaces, see corresponding remarks in $[8-10,28]$, e.g., where the exact asymptotic behavior is shown to be strongly dependent on the structure of the reaction kinetics and type of the moving front (sharp interface or not). This first step gives us additional motivation to reconsider from new perspectives our moving-interface approach to carbonation $[20,22]$.

Acknowledgements This work was partially supported by a grant through the special priority program DFG SPP1122 "Prediction of the course of physicochemical damage processes involving mineral materials". The author gratefully appreciates helpful discussions with S. Meier and M. Böhm (University of Bremen, Germany).
Open Access This article is distributed under the terms of the Creative Commons Attribution Noncommercial License which permits any noncommercial use, distribution, and reproduction in any medium, provided the original author(s) and source are credited.

\section{Appendix: Choice of scaling exponents}

We discuss the elimination procedure of the travelingwave solutions and of the other non-relevant situations which may occur as result of the scaling of the reaction front, see Sect. 3. To this end, we only solve systems of linear algebraic inequalities. The weak coupling between the mass-balance equation of moisture and the other mass balance equations allows us to proceed as in [3]:

Because of $\gamma=\frac{1}{p-1}$, the case (U3) is incompatible with both (V1) and (V2). Therefore all combinations containing (U3) have to be discarded.

If $v=\gamma$, then the latter assertion holds for (W3), too.

Obviously, in the case (V1) we have $\gamma=\frac{1}{p}$. This fact does not match the cases (U1), (U2) and (U3).

Gathering together these incompatibilities between the scaling exponents, we see that a successful configuration can only involve (U1), (U2), (V2), (W1) and (W2).

We note that there is a certain incompatibility between the cases (U2) and (V2) [and also (W2)]. Namely, from $\alpha-\beta+\gamma(p-1)-1=0$ and $\alpha-\beta+\gamma p-1=0$, we deduce that $\gamma=0$. This contradicts our choice of $\gamma$.

The triple (U1, V2, W1) is the successful combination. Notice that $\gamma \leq \frac{1}{p-1}$. From $\alpha-\frac{\gamma}{2}(p-1) \leq 0$ and $\gamma(p-1)=2 \beta$, we obtain that $\alpha \leq \frac{1}{2}$ and $\beta \leq \frac{1}{2}$.

\section{References}

1. Barenbaltt GI (1979) Similarity, self-similarity, and intermediate asymptotics. Consultants Bureau, NY

2. Bary B, Sellier A (2004) Coupled moisture-carbon dioxidecalcium transfer model for carbonation of concrete. Cem Concr Res 34:1859-1872

3. Bazant MZ, Stone HA (2000) Asymptotics of reactiondiffusion fronts with one static and one-diffusing reactant. Physica D 147:95-121

4. Bunte D (1994) Zum Karbonatisierungsbedingten Verlust der Dauerhaftigkeit von Außenbauteilen aus Stahlbeton. Dissertation, TU Braunschweig 
5. Cahyadi JH, Uomoto T (1993) Influence of environmental relative humidity on carbonation of concrete (mathematical modeling). Durability of building materials and components, vol 6 (Omiya, Japan), pp 1142-1151

6. Chaussadent T (1999) États de lieux et réflexions sur la carbonatation du béton armé. Rapport OA 29, LCPC, Paris

7. Do DD (1982) On the validity of the shrinking core model in non-catalytic gas-solid reactions. Chem Engng Sci 37:1477-1481

8. Emmerich H (2003) The diffuse interface approach in material sciences. Springer, Berlin

9. Fife PC (1988) Dynamics of internal layers and diffusive interfaces. SIAM, Philadelphia

10. Fila M, Souplet P (2001) Decay of global solutions, stability and blow-up for a reaction-diffusion problem with free boundary. Interfaces Free Bound 3:337-344

11. Hinch EJ (1991) Perturbation methods. Cambridge University Press, Cambridge

12. Houst Y, Wittmann FH, Roelfstra P (1983) A model to predict service life of concrete structures. Proc. Int. Conf. at Technische Akademie Esslingen, pp 181-186

13. Kropp J (1995) Relations between transport characteristics and durability. Performance criteria for concrete durability, RILEM Report 12 (Eds. J. Kropp, H.K. Hilsdorf) E and Fn Spon Editions, pp 97-137

14. Leger C, Argoul F, Bazant MZ (1999) Front dynamics during diffusion-limited corrosion of ramified electrodeposits. J Phys Chem B 103:5841-5851

15. Logan JD (2001) Transport modeling in hydrogeochemical systems. Springer, Berlin

16. Mainguy M (1999) Modèles de diffusion non-linéaires en millieux poreaux. Application a la dissolution et au séchage des matériaux cimentaires. These de doctorat, Ecole Normale des Ponts et Chaussées, Paris

17. Mainguy M, Coussy O (2000) Propagation fronts during calcium leaching and chloride penetration. ASCE J Engng Mech 3:252-257

18. Meier SA, Peter MA, Muntean A, Böhm M (2007) Dynamics of the internal reaction layer arising during concrete carbonation. Chem Engng Sci 162:1125-1137

19. Muntean A, Meier SA, Peter MA, Böhm M (2005) A note on the limitations of the use of accelerated concretecarbonation tests for service-life predictions. Berichte aus der Technomathematik, Tec. Report 05-04

20. Muntean A, Böhm M (2006) Dynamics of a moving reaction interface in a concrete wall. In: Free and moving boundary problems. theory and applications. Int series of num math, vol 154. Birkhäuser, Basel, pp 317-326
21. Muntean A, Böhm M (2006) Length scales in the concrete carbonation process and water barrier effect: a matched asymptotics approach. Berichte aus der Technomathematik, Tec. Report 07-06

22. Muntean A (2006) A moving-boundary problem: modeling, analysis and simulation of concrete carbonation. Cuvillier, Göttingen

23. Papadakis VG, Vayenas CG, Fardis MN (1989) A reaction engineering approach to the problem of concrete carbonation. AIChE J 35:1639

24. Saetta AV, Schrefler BA, Vitaliani RV (1993) The carbonation of concrete and the mechanism of moisture, heat and carbon dioxide flow through porous materials. Cem Concr Res 23(4):761-772

25. Schenkel A, Witter P, Stubbe J (1993) Asymptotics of solutions in an $A+B \rightarrow C$ reaction-diffusion system. Physica D 69:135-147

26. Sedov LI (1972) A course in continuum mechanics, vol. 2, Physical foundations and formulations of problems. Wolters-Nordoff, Groningen

27. Shampine LE, Gladwell I, Thompson S (2003) Solving ODEs with MATLAB. Cambridge University Press, Cambridge

28. Souplet P, Ghidousche H, Tarzia D (2001) Decay of global solutions, stability and blow-up for a reactiondiffusion problem with free boundary. Proc Am Math Soc 129:781-792

29. Steffens A (2000) Modellierung von Karbonatisierung und Chloridbildung zur numerischen Analyse der Korrosionsgefärdung der Betonbewehrung. Dissertation, Institut für Statik, Universität Braunschweig

30. Thiery M (2005) Modélisation de la carbonatation atmospherique des matériaux cimentaires. Rapport OA 52 (These de doctorat), LCPC, Paris

31. Thiery M, Baroghel-Bouny V, Dangla P, Villain G (2007) Numerical modeling of concrete carbonation based on durability indicators. CANMET/ACI Int. Conf. on Durability of Concrete, pp 765-780

32. Thiery M, Villain G, Dangla P, Platret G (2007) Investigation of the carbonation front shape on cementitious materials: effects of the chemical kinetics. Cem Concr Res 37:1047-1058

33. Yen A, Lin AL, Koo L, Vilensky B, Taitelbaum H, Kopelman R (1997) Spatiotemporal patterns and nonclassical kinetics of competing elementary reactions: chronium complex formation with xylenol orange in a capillary. J Phys Chem A 101:2819-2827 